\title{
Reflecting Cell Model for SAW Structures
}

\author{
Priyanka \\ Deenbandhu Chhotu Ram \\ University of Science and \\ Technology
}

\author{
Shiv Dutt Joshi \\ Indian Institute of Technology Delhi, \\ India
}

\author{
Brishbhan Singh Panwar \\ Indian Institute of Technology \\ Delhi, India
}

\begin{abstract}
The study presents three-port reflecting cell model for analyzing SAW structures employing strong electrode reflections. Basic objective is to analytically approximate all the p-matrix elements for a SAW structure in terms of reflection, transduction, and transmission strengths. This model is an approximate one and it does not take into account the effects of bulk wave excitation, diffraction, and propagation loss etc for convenience. This 3port reflecting cell model has been used to show that it is possible to

realize Infinite Impulse Response (IIR) filters on SAW devices, which can result in much smaller size as compared to very high order Finite Impulse Response (FIR) SAW filters for given specifications. Simulation results for this new 3-port reflecting cell model and IIR filter realization are presented for validation purpose.
\end{abstract}

\section{Introduction}

Present day SAW devices utilize reflections to have improved filter characteristics. Accurate analysis of SAW filters with reflections is much more complex than the analysis of SAW filters without reflections. The model that is often used for analyzing SAW transducers is the Coupling -of -Modes $(\mathrm{COM})$ model [1]. COM model is an approximate one and it represents the SAW IDT (which is discrete by nature) in terms of smoothly varying quantities governed by differential equations. Furthermore to relate the COM theory with transducer physics is not easy. COM theory represents wave parameters for COM, and then use COM for analysis of SAW structures [4]. Morgan has proposed the Reflective Array Modeling (RAM) [5] for SAW transducers that leads to a model more accurate and better justified than the COM. RAM can be used to analyze the reflections by cascading technique and calculate the p-matrix parameters $p_{11}, p_{12}, p_{22}$ analyses (to give $p_{13}, p_{23}$ ) RAM uses the method based on quasi-static analysis. Quasi-static methods are basically accurate for non-reflecting transducers, and therefore RAM can amplitudes by differential equations $[1,2]$ and assumes weak electrode reflections and narrow bandwidths. On the other hand the complex models utilizing Finite Element Method (FEM)/ Boundary Element Method (BEM) [3] can be quite good methods, but due to their complexity and large computation time it is common to use these models to calculate the required

provide accurate transduction $\left(p_{13}, p_{23}\right)$ only for SAW structures utilizing small electrode reflection coefficients.

SAW devices often include reflections for improved characteristics and strong reflections can be utilized for many additional advantages. The main attractiveness of 3-port reflecting cell model (RCM) presented in this paper is that it can be used to accurately model the reflection and transduction even for SAW structures utilizing strong reflections. On account of this RCM can be used to generate all p-matrix elements accurately for SAW unit cells utilizing large electrode reflection coefficients. Furthermore RCM retains the attractive simplicity of cascading methods in calculating all p-matrix elements of SAW structures. This model together with cascading formulas for $\mathrm{p}$-matrices demonstrates that rational transfer function on a SAW structure can be realized using two or more reflectors.

Realization of Infinite Impulse Response (IIR) transfer function on SAW devices leads to smaller size SAW filters as compared to high order Finite Impulse Response (FIR) realizations for same filter specifications. One can obtain IIR approximation for given FIR specifications using different techniques proposed already in literature [6-8].

\section{Preliminaries and Basic Assumptions}

Let $x(t)_{\text {be a signal and }} X(\omega)_{\text {be its Fourier transform. The }}$ $X(\omega) d \omega / 2 \pi$ can be understood as the amplitude of the exponential $\exp \{j \omega t\}$ in the signal $x(t)$ or the content of the frequency $\omega$ in the signal $x(t)$. Hence the expression of the signal in terms of its Fourier transform can be thought of as representation of the signal $x(t)$ as weighted sum 
of exponentials. As we make an assumption that resulting SAW filter is Linear-Time-Invariant (LTI), the output of an exponential input is also same exponential with amplitude and phase dictated by the frequency response of the resulting filter. This is because exponentials are eigen functions of LTI Systems. The approach for the analysis of SAW filters will be as follows: the objective is to get analytical expression for all the p-matrix elements by getting the output signals at three ports in terms of input signals for a unit cell. The p-matrices for InIDT (i. e. input IDT) and OutIDTs (i. e. output IDT) can be obtained by cascading the p-matrices for their unit cells. For a SAW IDT pparameters $p_{13}$ and/or $p_{23}$, which are the functions of frequency $(\omega=2 \pi \mathrm{f})$ can be assumed to give its transfer function.

Now we will briefly summarize various assumptions that will be taken and also various transformations will be modeled. The implications of these, wherever applicable, on the analysis are also discussed here:

When an exponential $\exp \{j \omega t\}$ travels from a point A to a point $\mathrm{B}$ a distance ${ }^{x_{0}}$, with a velocity 'vel', it undergoes a time lag of $t_{0}=x_{0} / v e l$. So the exponential received at point B becomes $\exp \left\{j \omega\left(t-t_{0}\right)\right\}$. Note that this time lag does not depend upon the frequency. Also, if we take into account attenuation say ' $c$ ', then it becomes $' c \exp \left\{j \omega\left(t-t_{0}\right)\right\}$ '. We will not consider attenuation ' $c$ ' in present analysis, as it is assumed to be negligible for convenience.

When an acoustic exponential wave strikes the finger pair with opposite polarity, current proportional to the finger overlap regenerates. This only constitutes the current flow by the forward moving acoustic wave. The second component, which contributes to induction to this current, is due to the acoustic wave coming from the following stage, i.e. the backward moving wave. Again the current induced by this component will be proportional to the finger overlap. This will get added to the component due to forward moving wave and a current proportional to the sum of these components will flow. This situation has been modeled as dependent current source. We assume that when an acoustic signal ' $a_{1}{ }^{\prime}$ reaches the finger pair with opposite polarity, current equal to ' $\alpha a_{1}{ }^{\prime}$ will get generated, where ' $\alpha$ ' is the transduction strength which is proportional to the overlap length of fingers with opposite polarity.

If the time taken by acoustic wave to travel through a unit cell (a distance equal to acoustic wavelength $\lambda$ ) is assumed to be ' $T$ ', then exponential will get modified by a multiplicative $\eta:$ Transmission coefficient of the reflector

$\alpha$ : Transduction coefficient

$\xi$ : acoustic to electrical signal conversion coefficient factor of $' \exp \{-j \omega T\}^{\prime}$, in traveling through one unit cell, as is clear from point 1 . We will consider this delay in signal as the unit delay, and will term this as $z^{-1}$. The frequency dependence of the phase shift is clearly incorporated in the above setting.

It is assumed that acoustic wave interacting with the reflector gets reflected from its center. When an acoustic wave reaches the reflector center, ' $\gamma$ ' times of the incident acoustic wave gets reflected and ' $\eta$ ' times get passes through the reflector. Here ' $\gamma$ ' is the reflection coefficient, and ' $\eta$ ' is the transmission coefficient of the reflector. The reflected wave gets coupled (i.e. added) to the backward moving wave, which is due to the acoustic wave coming from the following stage. The same is true for the acoustic wave coming from the reverse direction and interacting the reflector.

The parameters $\gamma, \eta$, and $\alpha$ have been taken to be constants but it can be seen from the analysis that they can be easily generalized to have frequency dependence.

\section{Three-port Reflecting Cell Model}

In this Section we present a novel approach to obtain p-matrix for a unit cell of SAW transducer with reflector. This model gives analytical expressions for all the p-matrix elements in terms of reflection strength, transduction strength, and transmission strength. The p-matrix for a SAW structure is obtained by cascading the p-matrices for the unit cells that it is comprised of. In the present analysis the reflection center is taken at the center of the reflector (center of the wide electrode of $3 \lambda_{0} / 8$ width), and the transduction center is taken at the center of the space between opposite polarity electrodes. DART structures [9] have been used for implementing the reflectivity in SAW structures, where three electrodes per unit cell are used. Narrow electrodes are of $\lambda_{0} / 8$ width, and reflectors are of $3 \lambda_{0} / 8$ the unit cell shown in Fig. 1 using three-port reflecting cell model.

The symbols used in present modeling are as follows:

$\omega=2 \pi f$, where $f$ is the frequency ( $\mathrm{Hz})$

$T$ : is the time required for SAW signal in traveling a unit cell.

$\gamma$ : Reflection coefficient of the reflector (wide electrode)

$G_{a: \text { Radiation conductance; }}$

$B_{a}$ :is unperturbed radiation susceptance and can be obtained by taking the Hilbert transform of $G_{a[10] \text {. }}$ 
$C_{T}$ : Capacitance per finger pair in $\mathrm{pF}$ that can be found by

standard electro acoustic methods [10].

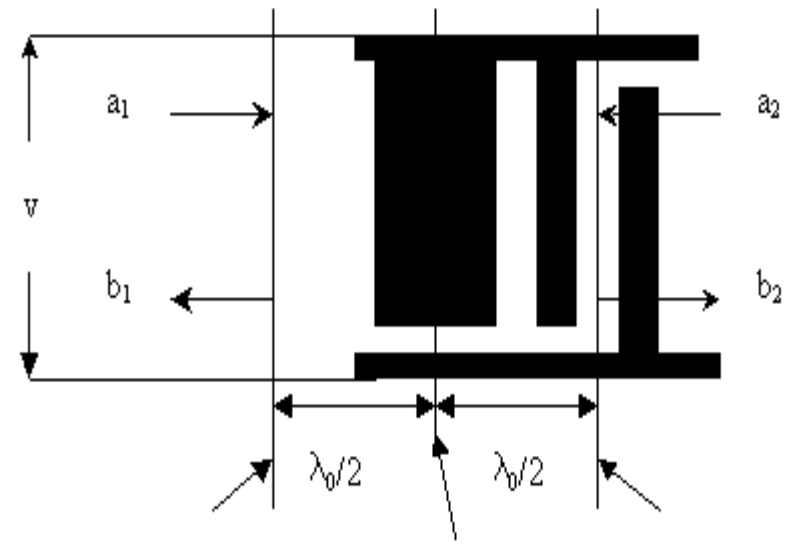

First reference plane

at $70 / 2$ distance from

Line through

reflection center

Second reference

plane going through

transduction center

Fig. 1. A SAW unit cell with reflector.

In Fig. 1 a1 and a2 (b1 and b2) are input (output) acoustic signals at port 1 and port 2 respectively. Let ' $\mathrm{v}$ ' is the applied voltage, and ' $\mathrm{i}$ ' is the current at port 3 , where port 1 and port 2 are acoustic ports and port 3 is electrical port. To get the required pmatrix elements we need to get expression for output signals in terms of input signals at three ports of a unit cell. According to the basic assumptions we can write from Fig. 1:

(1) 'al' is the input acoustic signal at port 1, signal ' $a_{1} \exp (-j \omega T / 2)$ ' will reach the reflection center, where it will split in transmitted and reflected signals from reflector:

(a) The reflected part ' $\left.a_{1} \exp (-j \omega T) \gamma\right)^{\prime}$ will reach the port 1 and will get added to outgoing acoustic signal from port 1.

$$
\text { Signal ' } a_{1} \exp (-j \omega T / 2) \eta^{\prime} \text { will get transmitted }
$$
through reflector and signal $' a_{1} \exp (-j \omega T) \eta^{\prime}$ will reach the transduction center and will lead to the ' $\xi a_{1} \eta \exp (-j \omega T)$ ' equivalent current flow in the bus bar. After that the signal ${ }^{\prime} a_{1} \exp (-j \omega T) \eta^{\prime}$ will get added to the outgoing acoustic signal at port 2.
(2) Signal ' $a_{2}{ }^{\prime}$ is the input acoustic signal at port 2, it will lead to ' $\xi a_{2}{ }^{\prime}$ current flow due to transduction center. After that signal ' $a_{2} \exp (-j \omega T / 2){ }^{\prime}$ will reach the reflection center, and it will get split in two parts, one is reflected part and other one is transmitted:

(a) Reflected part ' $a_{2} \exp (-j \omega T) \gamma^{\prime}$ will go to the transduction center and will lead to $\xi a_{2} \exp (-j \omega T) \gamma^{\prime}$ current flow, after that acoustic signal ' $a_{2} \exp (-j \omega T) \gamma^{\prime}$ will go out from port 2 of unit cell.

(b) The signal ' $a_{2} \exp (-j \omega T / 2) \eta^{\prime}$ will transmit through reflector, and ' $a_{2} \exp (-j \omega T) \eta^{\prime}$ will get added to outgoing acoustic signal at port 1 .

(3) On account of applied voltage ' $v$ ', the acoustic signal ' $\alpha v^{\prime}$ traveling in forward direction will get added to outgoing acoustic signal at port 2 . The acoustic signal ' $\alpha v^{\prime}$ will also travel in backward direction. The acoustic signal ' $\alpha v \exp (-j \omega T / 2)$ '

will reach the reflection center and it will split in two parts: 
(a) The reflected signal $\alpha v \exp (-j \omega T) \gamma^{\prime}$ will reach at the transduction center, leading to the ' $\xi \alpha v \exp (-j \omega T) \gamma^{\prime}$ current flow (however, magnitude of this regenerated current will be very small, and can be ignored) and then signal ' $\alpha v \exp (-j \omega T) \gamma^{\prime}$ will get added to outgoing acoustic signal at port 2 .

(b) Signal ' $\alpha v \exp (-j \omega T / 2) \eta^{\prime}$ will get transmitted through reflector and $' \alpha v \exp (-j \omega T) \eta^{\prime}$ will get added to outgoing acoustic signal at port1.

As a result of (i), (ii) and (iii) one can write:

$b_{1}=a_{1} \gamma \exp (-j \omega T)+a_{2} \eta \exp (-j \omega T)+\alpha v \eta \exp (-j \omega T)$ $b_{2}=a_{1} \eta \exp (-j \omega T)+a_{2} \gamma \exp (-j \omega T)+\alpha v+\alpha v \gamma \exp (-j$ $i=\xi a_{1} \eta \exp (-j \omega T)+\xi a_{2}+\xi a_{2} \gamma \exp (-j \omega T)+p_{33} v$

${ }_{\text {Where }} p_{33}=G_{a}(\omega)+j B_{a}(\omega)+j \omega C_{T}(\omega)_{[10], \quad \text { Eq }}$ (1) can be written in matrix form as:

$$
\left[\begin{array}{c}
b_{1} \\
b_{2} \\
i
\end{array}\right]=\left[\begin{array}{lll}
p_{11} & p_{12} & p_{13} \\
p_{21} & p_{22} & p_{23} \\
p_{31} & p_{32} & p_{33}
\end{array}\right]\left[\begin{array}{c}
a_{1} \\
a_{2} \\
v
\end{array}\right]
$$

Where p-matrix elements are defined as:

$$
\begin{aligned}
& p_{11}=\gamma \exp (-\mathrm{j} \omega \mathrm{T}) ; p_{12}=\eta \exp (-\mathrm{j} \omega \mathrm{T}) ; \\
& p_{13}=\alpha \eta \exp (-\mathrm{j} \omega \mathrm{T}) ; p_{21}=\eta \exp (-\mathrm{j} \omega \mathrm{T}) ; \\
& p_{22}=\gamma \exp (-j \omega T) ; p_{23}=\alpha+\alpha \gamma \exp (-j \omega T) ; \\
& p_{31}=\xi \eta \exp (-\mathrm{j} \omega \mathrm{T}) ; p_{32}=\xi+\xi \gamma \exp (-\mathrm{j} \omega \mathrm{T}) ; \\
& \mathrm{p}_{33}=G_{a}(\omega)+j B_{a}(\omega)+j \omega C_{T}(\omega)
\end{aligned}
$$

Expressing $\exp (-j \omega T)$ as unit delay and representing it as $z^{-1}$ ( basic assumption 3), we

Can write p-matrix elements in mathematical form as:

$$
\begin{aligned}
& p_{11}=\gamma \mathrm{z}^{-1} ; p_{12}=\eta \mathrm{z}^{-1} ; \\
& p_{13}=\alpha \eta \mathrm{z}^{-1} ; p_{21}=\eta \mathrm{z}^{-1} ; \\
& p_{22}=\gamma \mathrm{z}^{-1} ; p_{23}=\alpha+\alpha \gamma \mathrm{z}^{-1} ; \\
& p_{31}=\xi \eta \mathrm{z}^{-1} ; p_{32}=\xi+\xi \gamma \mathrm{z}^{-1} ; \\
& \mathrm{p}_{33}=G_{a}(\omega)+j B_{a}(\omega)+j \omega C_{T}(\omega)
\end{aligned}
$$

Or we can write:

$\left[\begin{array}{c}b_{1} \\ b_{2} \\ i\end{array}\right]=P\left[\begin{array}{c}a_{1} \\ a_{2} \\ v\end{array}\right]$, Where ' $P$, is the p-matrix for unit cell in Fig. 1. Using the fact that $p_{32}=-2 p_{23}$, we can write $\xi=-2 \alpha$

\section{Realization of IIR Transfer Function on SAW Structures:}

Realization of poles in a SAW structure with two or more reflecting electrodes results in rational transfer function for SAW structures. This should be noted that reflecting electrodes 968 Mjide electrodes having $3 \lambda 0 / 8$ width. Negligible reflections are assumed at narrow electrodes with $\lambda 0 / 8$ width. As a consequence of this no poles will be generated for a SAW structure having no wide electrode of $3 \lambda 0 / 8$ width. In this section we show that the pole can be generated in a SAW structure with two or more reflecting electrodes, or in other words rational transfer function will be realized for a SAW structure if there are two or more reflectors.

In order to get p-matrix for SAW structure, it is required to first obtain p-matrices for its unit cells and then cascade these pmatrices using cascading formulas as given by Abbott et. al. [1]. If p-matrix is obtained for a SAW structure having two or more reflectors, a rational transfer function will be realized. This shows that it is possible to realize IIR filter on a SAW device.

One of the most important factors in realization of IIR transfer function on SAW devices is the realization of a proper second order denominator polynomial in rational transfer function for a SAW structure. If one could achieve this then it can be said that realization of any rational transfer function is possible on a SAW device. In this section we show that a proper second order denominator polynomial in a rational transfer function given by p-parameters $p_{13}$, (and/or $p_{23}$ ) can be realized. In particular we show that the SAW structure shown in Fig. 2 realizes a proper second order denominator polynomial for p-matrix

elements $p_{13 \text { (and/or }} p_{23}$ ). The p-matrix for this SAW structure can be obtained by using the RCM modeling and cascading formulas as already discussed. The analytic expressions for p-matrix elements for structure in Fig.2 are as follows: 


$$
\begin{aligned}
& p_{11}=\frac{\gamma z^{-1}+\left(-3 \gamma^{3}+\gamma \eta^{2}\right) z^{-2}+\left(2 \gamma^{5}-3 \gamma^{3} \eta^{2}+\gamma^{5}+\gamma \eta^{4}\right) z^{-3}+\left(-\gamma^{7}+2 \gamma^{5} \eta^{2}-\gamma^{3} \eta^{4}\right) z^{-4}}{\left(1-\gamma^{2} z^{-1}\right)\left[1-2 \gamma^{2} z^{-1}+\left(\gamma^{4}-\gamma^{2} \eta^{2}\right) z^{-2}\right]} \\
& p_{12}=\frac{\eta^{3} z^{-2}}{1-2 \gamma^{2} z^{-1}+\left(\gamma^{4}-\gamma^{2} \eta^{2}\right) z^{-2}} \quad p_{13}=\frac{-\alpha \eta^{3} z^{-2}}{1-2 \gamma^{2} z^{-1}+\left(\gamma^{4}-\gamma^{2} \eta^{2}\right) z^{-2}} \\
& p_{23}=\frac{\alpha\left[1+\left(-\gamma-2 \gamma^{2}\right) z^{-1}+\left(2 \gamma^{3}+\gamma^{4}-\gamma^{2} \eta^{2}-\gamma \eta^{2}\right) z^{-2}+\left(-\gamma^{5}+2 \gamma^{3} \eta^{2}-\eta^{4} \gamma\right) z^{-3}\right.}{1-2 \gamma^{2} z^{-1}+\left(\gamma^{4}-\gamma^{2} \eta^{2}\right) z^{-2}}
\end{aligned}
$$

Where $z=\exp (-j \omega T)$, is unit delay, which is equal to the delay on account of traveling a unit cell or a length $\lambda$ the acoustic wavelength, and all other parameters are as already defined.

$p_{33}$ can be calculated by the method used in RAM [10] as explained earlier
Realization of a proper second order denominator polynomial in p-matrix elements $p_{13}$ and $p_{23}$ establishes the claim that it is possible to realize a pole on SAW device.
First reference plane of first unit cell

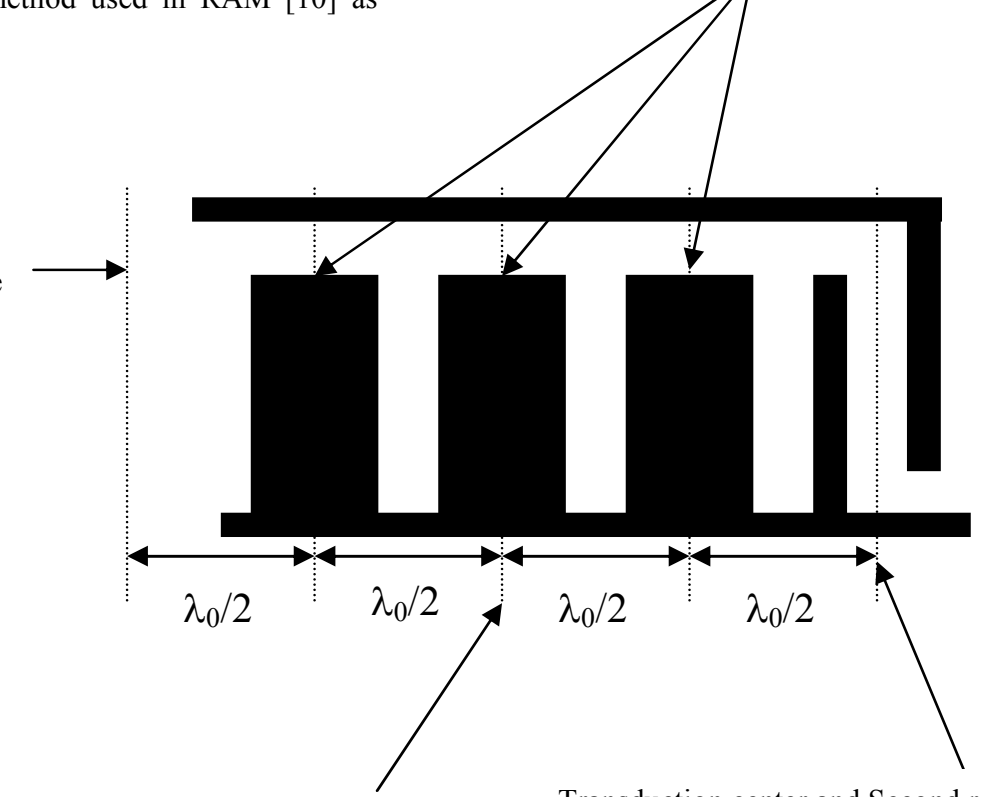

Transduction center and Second ref plane of second unit cell
First reference plane of second unit cell and second reference plane of first unit cell

\section{Reflectors}




\section{Simulation and Validation of Results}

In order to validate RCM model presented in this paper the results given by RCM model were compared with that given by COM model. As the model is used to generate the p-matrix, all the p-matrix elements were generated using RCM and COM model for a number of SAW transducers. It has been shown by simulation that all the corresponding elements of p-matrices predicted by two models for SAW transducers on Quartz substrate and two normalized film thicknesses shows excellent match. However, results only for p-parameters $p_{13}$ and $p_{\mathbf{2 8}}$ are given here. It has to be noted that basic objective of simulation results presented here is to compare the results given by two models COM and RCM.
Transducer 1: This transducer is having transduction and reflection weighting coefficients as that obtained for IDT 1 of SAW filter 1 in Reference [11]. This sequence of transduction and reflection weighting coefficients has been analyzed by COM and RCM modeling with two different normalized film thickness ratios $(\mathrm{h} / \lambda)$ equal to $1.16 \%$ and $3.75 \%$ for ST-quartz substrate. The purpose to choose these two normalized film thickness ratios was to compare the p-parameters for moderate reflections $(\mathrm{h} / \lambda=1.16 \%)$ and strong reflections $(\mathrm{h} / \lambda=3.75 \%)$.

The magnitude of p-parameters ${ }^{p_{10}}$ and ${ }^{N_{2}}$ for this transducer given by RCM and COM under unmatched condition are given in Fig. 3 to Fig. 6. It can be seen from simulation results that the p-parameters for two different normalized film thicknesses $(\mathrm{h} / \lambda)$ are quite different due to change in reflectivity and other parameters. But the differences predicted by RCM and COM are quite similar.

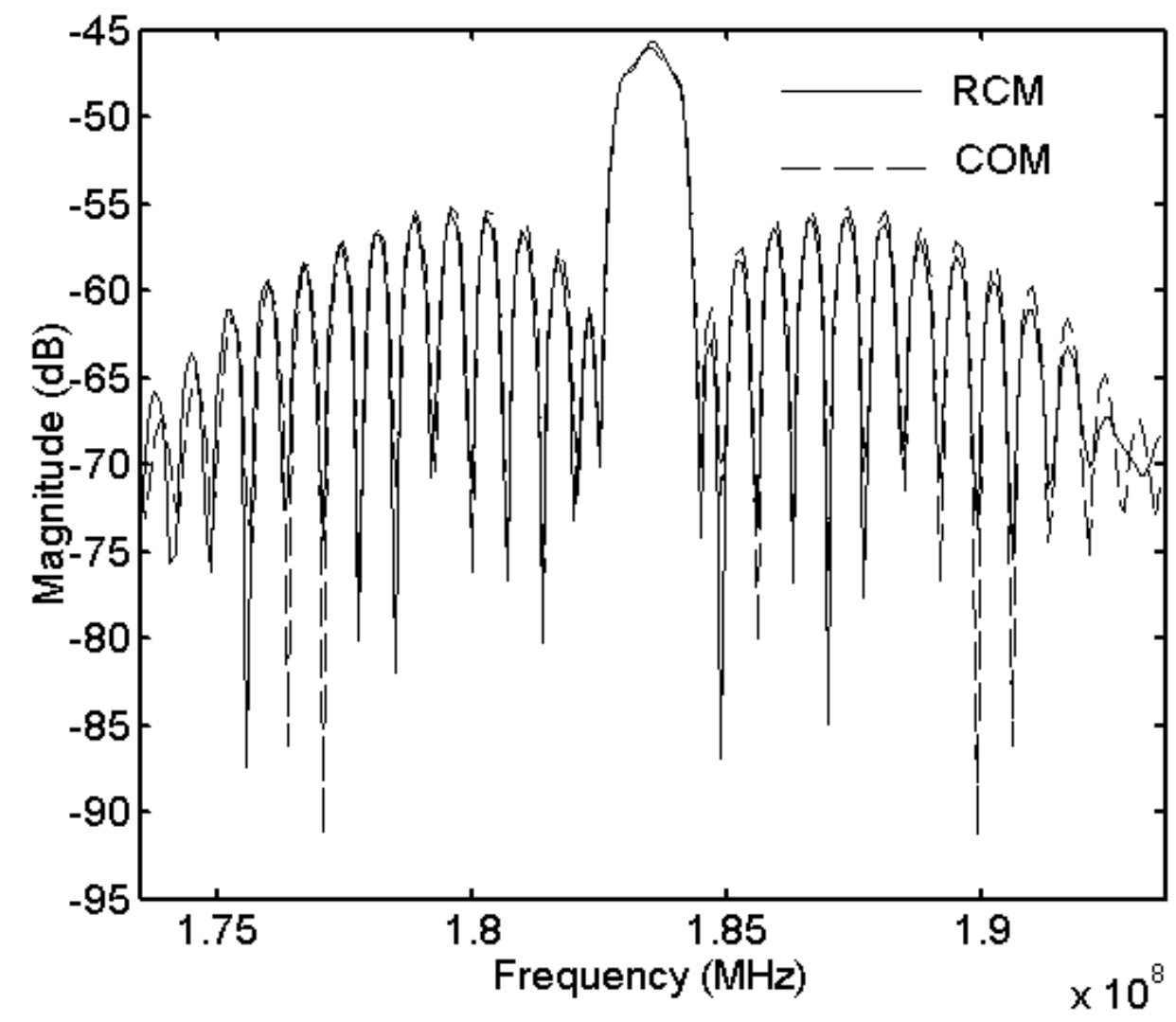

Fig. 3 simultaneous plots of $p_{13}$ given by present model (RCM) and COM model for transducer 1 with (h/ $\lambda$ )

equal to $1.16 \%$. 


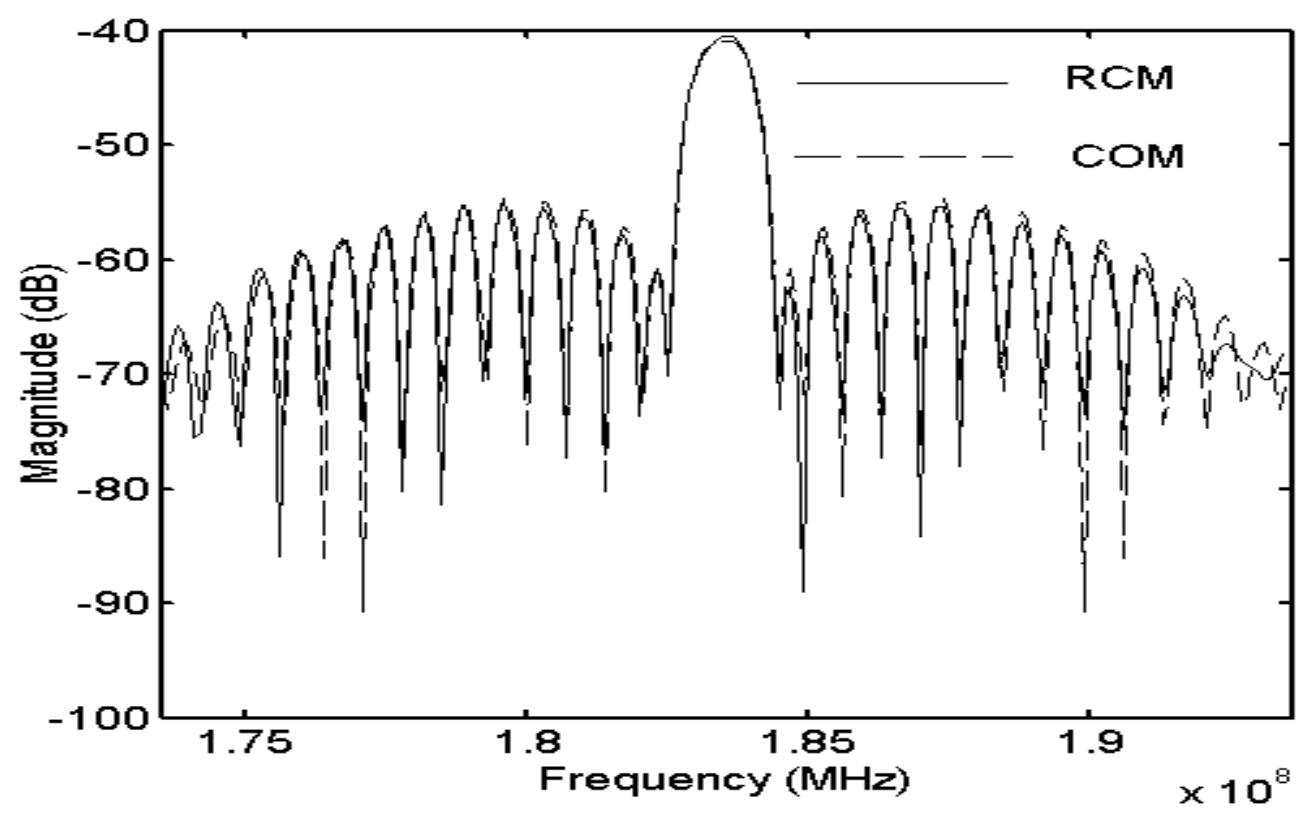

Fig. 4 simultaneous plots of $p_{23}$ given by present model (RCM) and COM model for transducer 1 with $(h / \lambda)$ equal to $1.16 \%$.

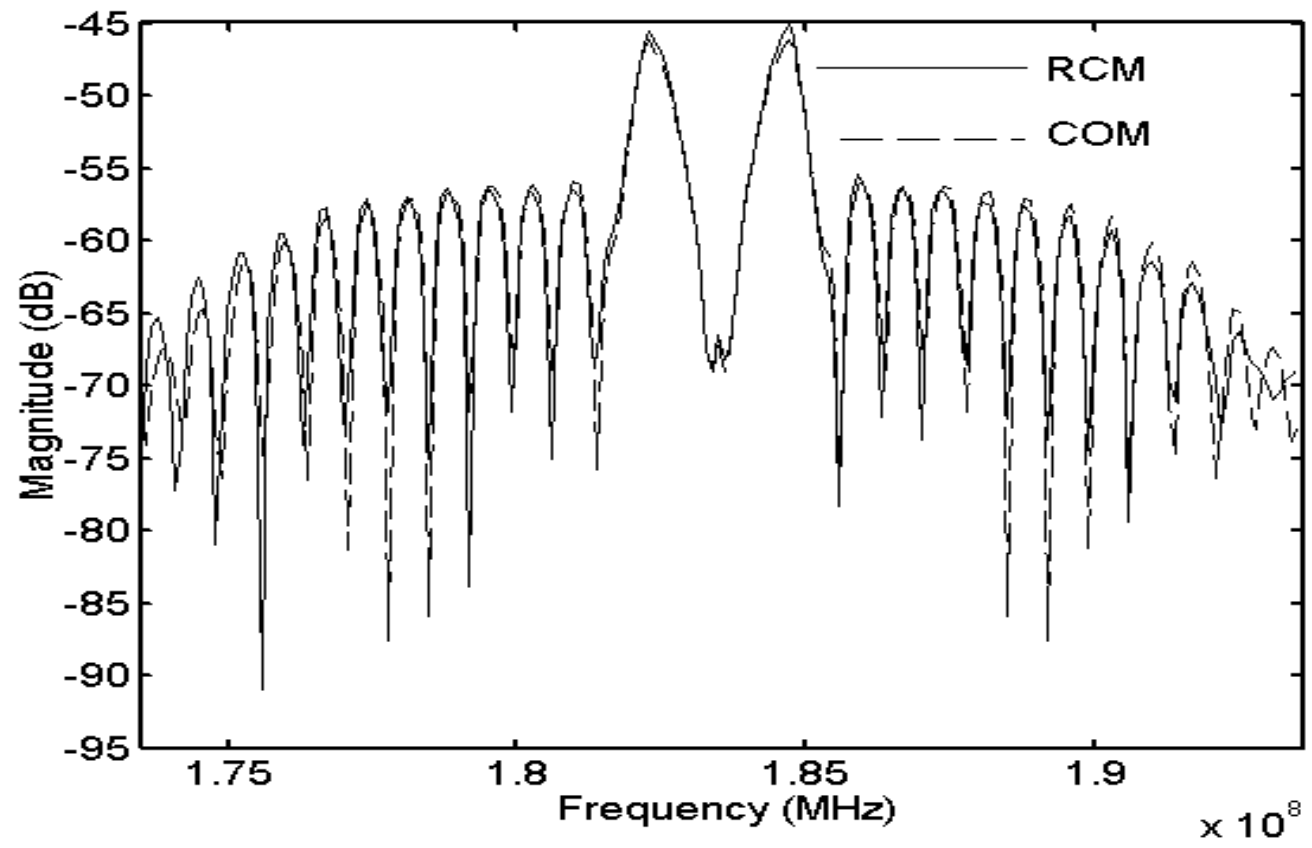

Fig. 5 simultaneous plots of $p_{13}$ given by present model (RCM) and COM model for transducer 1 with $(h / \lambda)$ equal to $3.75 \%$. 


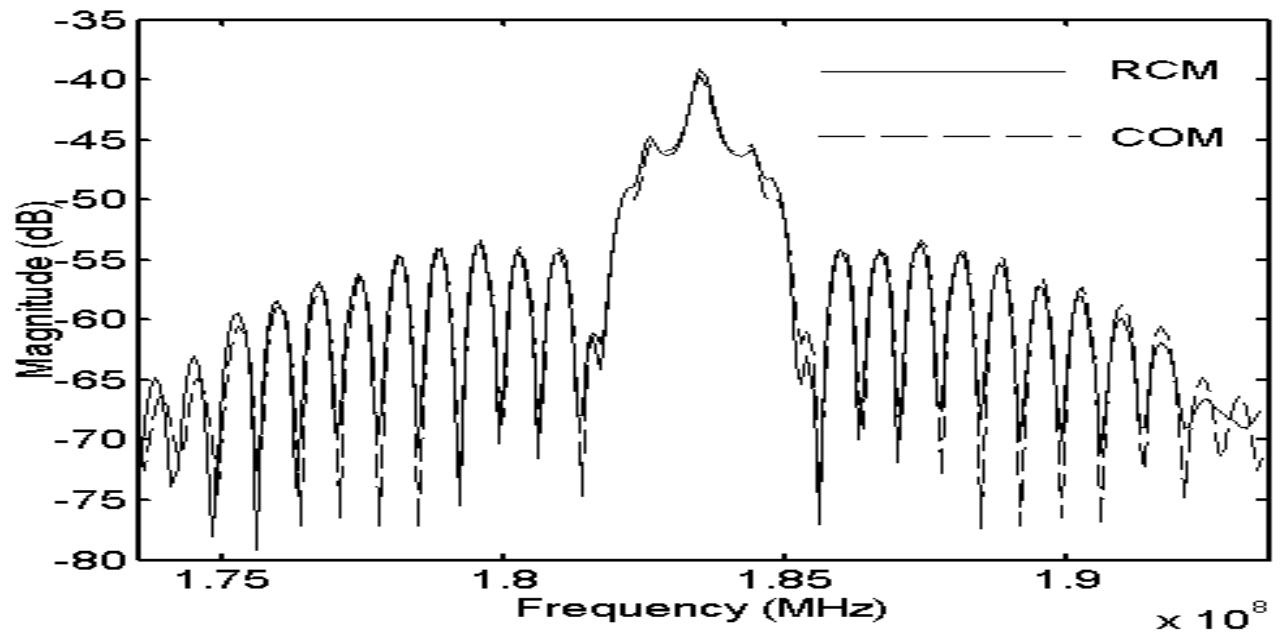

Fig. 6 simultaneous plots of $p_{23}$ given by present model (RCM) and COM model for transducer 1 with (h/ $\lambda$ )

equal to $3.75 \%$.

RCM model has been compared with COM for all p-matrices for other transducers of Reference [11] and for other different SAW structures. RCM has shown excellent match with COM for all other SAW transducers also.

To validate the model for simple bi-directional transducers (with no reflecting structures), the results for $\mathrm{p}$-parameters for transducer 2 of SAW filter 2 in Reference [11] with same transduction coefficients and zero reflection coefficients have been compared with COM. For bi-directional transducers one can predict the original transfer function by simply taking the Fourier transform of transduction coefficients. However, loss cannot be predicted by this original transfer function. The three transfer functions, the Original transfer function (given by transduction coefficients), $p_{13}$ predicted by RCM, and $p_{13}$ predicted by COM has been plotted simultaneously in Fig.7, where loss in original transfer function has been matched with that predicted by $\mathrm{COM}$ (under unmatched conditions) by dividing the original transfer function with suitable real positive value determined by the loss predicted by COM under unmatched conditions. This can be seen from fig. 7 that there is perfect match of three transfer functions for bidirectional transducers.

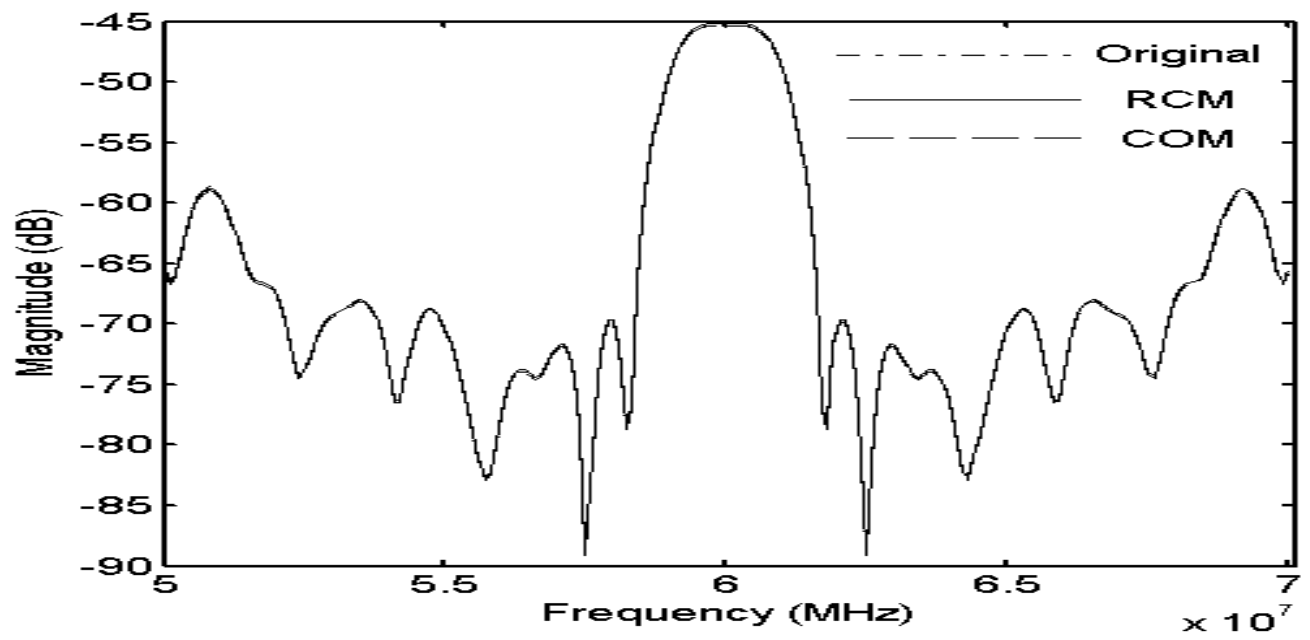

Fig. 7 simultaneous plots of $p_{13}$ given by present model (RCM), COM model, together with original transfer function (with loss matched to that given by COM model under unmatched conditions) for transducer 2 , with zero reflection coefficients. 


\section{CONCLUSION}

The 3-port reflecting cell model (RCM) has been presented which can be used to analyze the SAW structures with/without reflecting electrodes. This model can be used to get analytical expressions of all the p-matrix elements in terms of reflection, transduction and transmission strengths. The basic advantage of this model is that it can be used to obtain all the p-matrix elements accurately even for the SAW structures utilizing strong reflection coefficients. Furthermore this model retains the computational convenience in computing all the p-matrix elements. Simulation results show excellent match for all the pparameters given by RCM and COM for SAW structures on quartz with different normalized film thickness ratios $(h / \lambda)$.

However, results for p-matrix elements $p_{13}$ and $p_{23}$ are shown only, as this represents the transfer function. Using RCM together with cascading formulas for p-matrices, it has been shown that IIR transfer function can be realized on a SAW structure having two or more reflectors. Realization of proper second order denominator polynomial on a SAW structure has been shown, that establishes the claim that it is possible to realize any rational transfer function on a SAW structure.

\section{REFERENCES:}

[1] B. P. Abbott, C. S. Hartmann, and D. C. Malocha, "A Coupling-of-Modes analysis of chirped transducers containing reflective electrode geometries", in Proc. IEEE Ultrason. Symp, 1989, pp. 129-134.

[2] D. P. Chen and H. A. Haus, "Analysis of metal-strip SAW gratings and transducers", IEEE Trans. Sonics Ultrason. 32, pp. 395-408, 1985.

[3] N. Finger, G. Kovacs, J. Schoberl, and U. Langer, "Accurate FEM/BEM simulation of surface acoustic wave filters”, in Proc. IEEE Ultrason. Symp., 2003, pp. 1680-1685.
[4] K. Hashimoto and M. Yamaguchi, "Derivation of Coupling-of-Modes parameters for SAW device analysis by means of boundary element method", in Proc. IEEE Ultrason. Symp., 1991, pp. 21-26.

[5] D. P. Morgan, "Reflective array modeling for SAW transducers", in Proc. IEEE Ultrason. Symp., 1995, pp. 215-220.

[6] F. Brophy and A. C. Salazar, "Considerations of the Pade approximant technique in the synthesis of recursive digital filters", IEEE Trans. Audio Electroacoust., vol. AU-21,pp. 500505, Dec. 1973.

[7] L. Weiss and R. N. McDonough, "Prony's method, ztransforms, and Pade approximations", SIAM Rev., vol. 5, no. 2, pp. 145-149, Apr. 1963.

[8] C. T. Mullis and R. A. Roberts, "The use of secondorder information in the approximation of discrete-time linear systems", IEEE Trans. Acoust., Spe., Sig., Proces., vol. 24, no. 3, pp. 226-238, June 1976.

[9] T. Kodama, H. Kawabata, Y Yashuhara, \& H. Sato, "Design of low -loss SAW filters Employing Distributed Acoustic Reflection Transducers", Proc. IEEE Ultrason. Symp., pp. 59-64, 1986.

[10] D. P. Morgan, "Reflective array modeling for reflective and directional SAW transducers" IEEE Trans. Ultrason., Ferroelect., Freq. Contr., vol. 45, pp. 152-157, 1998.

[11] Priyanka, Shiv Dutt Joshi, and Brishbhan Singh Panwar "An Iterative Approach to Design a SAW Filter having Desired Response", IEEE Transactions on Ultrasonics, Ferroelectrics, and Frequency Control, August' 2005, vol. 52, no.

8 ,

pp.

1422. 